\title{
Urodynamic Findings in Pediatric Spastic Cerebral Palsy: Retrospective Study From a Single Center
}

\section{Wenbin Jiang}

Shanghai Jiao Tong University

Huizhen Sun

Shanghai Children's Hospital, Shanghai Jiao Tong University

\section{Baojun Gu}

Shanghai Sixth People's Hospital

\section{Qijia Zhan}

Shanghai Children's Hospital, Shanghai Jiao Tong University

\section{Min Wei}

Shanghai Children's Hospital, Shanghai Jiao Tong University

\section{Sen Li}

Shanghai Children's Hospital, Shanghai Jiao Tong University

\section{Fang Chen}

Shanghai Sixth People's Hospital

\section{Bo Xiao ( $\nabla$ xiao977@hotmail.com )}

Shanghai Children's Hospital, Shanghai Jiao Tong University

\section{Research Article}

Keywords: Cerebral Palsy, Urodynamics, Urinary Bladder, Neurophysiology

Posted Date: November 10th, 2021

DOI: https://doi.org/10.21203/rs.3.rs-990667/v1

License: (c) (i) This work is licensed under a Creative Commons Attribution 4.0 International License. Read Full License 


\section{Abstract}

Objective To investigate the urodynamic study (UDS) result in pediatric patients with spastic cerebral palsy (CP).

Material and methods Medical records of CP with pre-operative UDS results underwent selective dorsal rhizotomy (SDR) from Jan. 2020 to May. 2021 were retrospectively reviewed.

Results Fifty-seven cases with spastic CP were included in the study. Among these cases, 46 were ambulatory and 11 were non-ambulatory. Average gross motor function measure - 66 (GMFM - 66) score was $62.16 \pm 11.39$. Reduced bladder capacity was seen in $49.12 \%$ of these cases and cases with lower GMFM - 66 score had a higher incidence rate of having low bladder capacity $(p<0.01)$. Detrusor overactivity (DO) was shown in $33.33 \%$ of patients. Cases with younger age had higher prevalence of DO $(p<0.05)$. Meanwhile, more non-ambulant patients had DO $(p<0.05)$. Increased post-voiding residual (PVR) was seen in $21.05 \%$ of cases. Those with higher average threshold in sphincterassociated input spinal nerve roots (rootlets) had higher rate of having abnormal PVR $(p<0.05)$.

Conclusion Abnormal UDS results were prevalent in pediatric spastic CP. Motor function, age and threshold of their sphincter-associated spinal nerve rootlets were related to the abnormal UDS results.

\section{Introduction}

Urodynamic study (UDS) is an effective quantitative measure to assess urinary function, and it has been clinically applied for many years ${ }^{1}$. Micturition dysfunction is commonly seen in children with spastic cerebral palsy (CP) ${ }^{2,3}$.

Previous studies usually put their focuses on the impact of spasticity on motor function development in these patients. Much fewer literatures investigated micturition function using UDS. Most of the existing researches which applied UDS were restricted by their sample size, making it difficult to conduct cohorts analysis ${ }^{4,5}$. Due to technical limitations, it was difficult to investigate studies to find the relation between electrophysiological characteristics in spinal circuits and micturition dysfunction in such patients. Selective dorsal rhizotomy (SDR) via single level approach for cases with spastic CP requires to identify each root (rootlet) of cauda equina at L2 level using trigger EMG interpretation, in order to figure out sensory roots (rootlets) associated with lower limb spasticity. Therefore, it provides an effective method to record neurophysiological data for each root (rootlet) in caudal equina during surgery. When such data combined with UDS results, it might help researchers to learn more about the underlying mechanisms of such disorder ${ }^{6,7}$.

The purpose of the current study aimed to improve our understandings of characteristics of abnormal micturition in children with spastic CP.

\section{Material And Methods}

Medical records of pediatric patients with a diagnosis of spastic CP operated on using SDR at our center from Jan. 2020 to May. 2021 were retrospectively reviewed. Indication of SDR was previously documented in our published paper, which was not discussed in this article ${ }^{6}$. All included cases had pre-op UDS examination before SDR. The diagnosis of spastic CP was made by our multi-disciplinary team including neurosurgeons, neurologists and physical therapists. Clinical data including demographics, urodynamic results, relevant evaluation records, as well as intra-operative neurophysiological data of included cases were taken from the Database of Pediatric Cerebral Palsy Patients in our hospital.

\section{Motor function assessment}


Motor function of our cases was assessed using gross motor function classification system (GMFCS) and gross motor function measure-66 (GMFM-66). GMFCS is a five-level classification system depicting functional abilities in sitting, walking and need for assistive devices such as walkers or wheelchairs. Children in Level I can generally walk without restrictions but tend to be limited in some more advanced motor skills and those in Level V are usually very limited in their ability to move themselves around ${ }^{8}$. GMFM- 66 is a standardized observational instrument designed to measure change in gross motor function over time ${ }^{9}$.

\section{UDS}

The UDS test was done based on the standardization of the International Children's Continence Society (ICCS) ${ }^{10}$. All tests were done with Ellipse (ANDROMEDA MedizinischeSysteme GmbH, Wallbergstr.5, 82024 Taufkirchen/Potzham Germany). UDS was performed with slow fill water cystometry using a 6-Fr double lumen UDS catheter, with patient keeping supine without sedation and a rectal catheter was inserted into the anal to measure abdominal pressure. The infusion rate of saline was $10 \%$ estimated bladder capacity (EBC) per minute, and age-related bladder capacity was calculated with the equation (age +1$) \times 30 \mathrm{ml}$ in patients younger than 11 years old and a minimum capacity of $350 \mathrm{ml}$ was taken as the baseline for patients aged 12 years and older ${ }^{11}$. Filling of saline was discontinued when the child indicated fullness or when significant leakage occurred. Clinical testing was followed by sonography of kidneys when the bladder was empty and residual urine was measured twice. The lowest residual urine measurement was used for the evaluation.

Total bladder capacity was defined as the total volume of water infused until a filling end point. Small bladder was defined as bladder capacity < 65\% EBC ${ }^{12}$. Involuntary detrusor pressure increases over $15 \mathrm{~cm} \mathrm{H} 20$ was considered as an overactive detrusor contraction (Figure 1) ${ }^{13}$. Post-voiding residual (PVR) $\geq 20 \mathrm{ml}$ were defined as elevated according to the definition of ICCS ${ }^{14}$. Other UDS parameters such as bladder compliance were also included. Bladder compliance was measured with the algorithm $\Delta \mathrm{V} / \Delta \mathrm{P}$ det. $\Delta \mathrm{V}$ is the mean volumetric capacity and $\Delta \mathrm{P}$ det is the detrusor pressure rise. Children with bladder compliance of $15 \mathrm{~mL} / \mathrm{cm} \mathrm{H} 2 \mathrm{O}$ and higher were defined as normal ${ }^{15}$.

\section{Intra-operative neurophysiology}

All patients included in this study underwent SDR using modified intra-operative neurophysiologic protocol ${ }^{16}$. Surgery was performed under general anesthesia with minimum alveolar concentration (MAC) of sevoflurane inhalation at 0.5 and maintenance of body temperature between 36.0 and $37.0^{\circ} \mathrm{C}$. A total of 15 channels, including external anal sphincter (EAS) were neuro-physiologically monitored. All spinal nerve roots (rootlets) at L2 level were stimulated. Cadwell-Cascade Elite monitoring system was used in our center. Based on the protocol, all sensory roots (rootlets) on which single pulse stimulation first evoked responses in channel of EAS were considered sphincter-associated input roots (rootlets) (SAIR). The electrical current given to SAIR, which could elicit responses reaching around $20 \mu \mathrm{V}$ in channel of EAS was defined as its threshold (Figure 2). Due to the fact that usually 10-20 roots (rootlets) could match the definition of SAIR based on the protocol in each case, an average of electrical threshold in all these rootlets in each case was our focus in the current study.

\section{Statistical analysis}

Data was demonstrated as "mean \pm SD" if it was normal distribution and as "median (Q1, Q3)" if not. For categorical data, statistical comparison was done using Chi-Square test, and continuity correction was used whenever appropriate. Independent t-test was used for statistical analysis of data fitting normal distribution, and Mann-Whitney U test was used for data didn't meet normal distribution. Statistical significance was assumed if $p<0.05$. 


\section{Result}

A total of 57 cases were included in the current study, among which 44 (77.19\%) were boys. The mean age at the time of SDR was $6.73 \pm 2.84$ with range from 3 to 17 years old. The most two common etiologies of CP were asphyxia (56.14\%) and premature (24.56\%). In cases included, 8 (14.04\%), 39 (68.42\%) and 10 (17.54\%) were spastic hemiplegic, diplegic and quadriplegic, respectively. Among our cases, 46 (80.70\%) were ambulatory (GMFCS level $\nabla$ to $\nabla)$ whilst 11

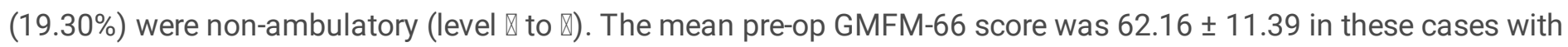
range of 29.96 to 82.99. Detailed demographic information was shown in Table 1. All patients except one (3 years old who required diaper) could go to toilet to void/defecate spontaneously. 
Table 1

Demographic details of 57 cases included in this study

\begin{tabular}{|c|c|}
\hline Characteristics & No., \% \\
\hline \multicolumn{2}{|l|}{ Gender } \\
\hline Boy & $44,77.19 \%$ \\
\hline Girl & $13,22.81 \%$ \\
\hline Age at surgery, year (mean \pm SD) & $6.73 \pm 2.84$ \\
\hline \multicolumn{2}{|l|}{ Etiology of spasticity } \\
\hline Premature & $14,24.56 \%$ \\
\hline Asphyxia & $32,56.14 \%$ \\
\hline Unknown & $11,19.30 \%$ \\
\hline \multicolumn{2}{|l|}{ Spastic type } \\
\hline Hemiplegia & $8,14.04 \%$ \\
\hline Diplegia & $39,68.42 \%$ \\
\hline Quadriplegia & $10,17.54 \%$ \\
\hline \multicolumn{2}{|l|}{ GMFCS level } \\
\hline Level $\nabla$ & $4,7.02 \%$ \\
\hline 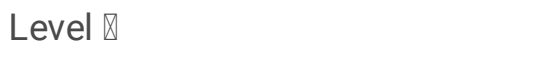 & $20,35.09 \%$ \\
\hline Level $\mathbb{Z}$ & $22,38.60 \%$ \\
\hline Level $\mathbb{Z}$ & $10,17.54 \%$ \\
\hline 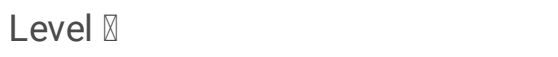 & $1,1.75 \%$ \\
\hline GMFM - 66 score (mean \pm SD) & $62.16 \pm 11.39$ \\
\hline \multicolumn{2}{|l|}{ Bladder/bowel symptoms } \\
\hline Enuresis & $4,7.02 \%$ \\
\hline Urge urinary incontinence & $17,29.82 \%$ \\
\hline Frequency & $7,12.28 \%$ \\
\hline Urgency & $5,8.77 \%$ \\
\hline Normal voiding & $27,47.37 \%$ \\
\hline Constipation & $17,29.82 \%$ \\
\hline Normal defecation & $40,70.17 \%$ \\
\hline \multicolumn{2}{|l|}{ Urodynamic outcomes } \\
\hline Overactive Bladder & $19,33.33 \%$ \\
\hline \multicolumn{2}{|c|}{ GMFCS: Gross Motor Function Classification System } \\
\hline GMFM - 66: Gross Motor Functio & asurement - 66 \\
\hline
\end{tabular}




\begin{tabular}{|lc|}
\hline Characteristics & No., $\%$ \\
\hline Small bladder capacity & $28,49.12 \%$ \\
\hline Increased post-voiding residual & $12,21.05 \%$ \\
\hline Normal & $17,29.82 \%$ \\
\hline GMFCS: Gross Motor Function Classification System \\
\hline GMFM - 66: Gross Motor Function Measurement - 66 \\
\hline
\end{tabular}

Thirty patients (52.63\%) had one or more lower urinary tract symptoms (LUTS). Urge urinary incontinence was the most common symptom presented in 17 cases (29.82\%), in which 14 had daytime incontinence nearly once a week, whilst the others once a day. Frequency (> 7 times per day), urgency and enuresis were observed in 7 (12.28\%), 5 (8.77\%) and 4 (7.02\%) cases, respectively. Besides with LUTS, 17 (29.82\%) cases suffered from bowel symptoms (constipation).

Abnormal UDS findings were revealed in 40 cases (70.18\%). In 57 cases included in the current study, bladder capacity ranged from 55 to $450 \mathrm{ml}$, and the average figure was $60.63 \%$ of estimated bladder capacity (EBC). Twenty-eight (49.12\%) cases had a bladder capacity less than 65\% EBC. Detailed statistical comparisons were listed in Table 2. Significant difference was found in the GMFM-66 score when comparing cases with normal/small bladder capacity. It was observed that cases with GMFM- 66 score less than 70.22 were more likely having small bladder capacity. The cutoff point (70.22) was calculated with receiver operating characteristic (ROC) curve with area under curve (AUC) equaling 0.67 (Figure 3-A, $\mathrm{p}<0.05$ ). 
Table 2

Comparisons between cases with and without UDS abnormalities.

\begin{tabular}{|c|c|c|c|c|c|c|c|c|c|}
\hline \multirow[t]{2}{*}{ Characteristics } & \multicolumn{3}{|c|}{ Bladder Capacity } & \multicolumn{3}{|c|}{ Detrusor activity } & \multicolumn{3}{|l|}{ PVR } \\
\hline & Decreased & Normal & $\begin{array}{l}p \\
\text { value }\end{array}$ & Overactivity & Normal & $p_{\text {value }}$ & Increased & Normal & $\begin{array}{l}p \\
\text { value }\end{array}$ \\
\hline \multicolumn{10}{|l|}{$\begin{array}{l}\text { Categorical } \\
\text { data }\end{array}$} \\
\hline \multicolumn{10}{|l|}{ Gender (n) } \\
\hline Boy & 20 & 24 & 0.31 & 16 & 28 & $0.58^{*}$ & 9 & 35 & 0.85 \\
\hline Girl & 8 & 5 & & 3 & 10 & & 3 & 10 & \\
\hline \multicolumn{10}{|l|}{ GMFCS (n) } \\
\hline Level $\rrbracket-\rrbracket$ & 21 & 25 & $0.46^{*}$ & 12 & 34 & $0.04^{*}$ & 9 & 37 & $0.88^{*}$ \\
\hline Level $\rrbracket-\rrbracket$ & 7 & 4 & & 7 & 4 & & 3 & 8 & \\
\hline \multicolumn{10}{|l|}{$\begin{array}{l}\text { Quantitative } \\
\text { data }\end{array}$} \\
\hline \multicolumn{10}{|l|}{ Age (years) } \\
\hline Median & 6.50 & 5.00 & 0.26 & 5.00 & 6.25 & 0.03 & 6.50 & 6.00 & 0.12 \\
\hline (Q1, Q3) & $\begin{array}{l}(5.00 \\
8.50)\end{array}$ & $\begin{array}{l}(4.00 \\
9.00)\end{array}$ & & $(4.00,8.00)$ & $\begin{array}{l}(5.00 \\
9.00)\end{array}$ & & $\begin{array}{l}(5.50 \\
10.75)\end{array}$ & $\begin{array}{l}(4.50 \\
8.50)\end{array}$ & \\
\hline $95 \% \mathrm{Cl}$ & $5.50-8.00$ & $\begin{array}{l}4.50- \\
6.50\end{array}$ & & $4.00-8.00$ & $\begin{array}{l}5.50- \\
8.50\end{array}$ & & $\begin{array}{l}5.50- \\
11.50\end{array}$ & $\begin{array}{l}5.00- \\
8.00\end{array}$ & \\
\hline \multicolumn{10}{|l|}{$\begin{array}{l}\text { GMFM-66 } \\
\text { score }\end{array}$} \\
\hline Mean \pm SD & $\begin{array}{l}58.20 \pm \\
10.27\end{array}$ & $\begin{array}{l}65.98 \\
\pm \\
11.46\end{array}$ & $<0.01$ & $\begin{array}{l}59.95 \pm \\
13.27\end{array}$ & $\begin{array}{l}63.26 \\
\pm \\
10.50\end{array}$ & 0.31 & $\begin{array}{l}59.46 \pm \\
8.97\end{array}$ & $\begin{array}{l}62.88 \\
\pm \\
12.05\end{array}$ & 0.36 \\
\hline $95 \% \mathrm{Cl}$ & $\begin{array}{l}54.22- \\
62.18\end{array}$ & $\begin{array}{l}61.62- \\
70.34\end{array}$ & & $\begin{array}{l}53.56- \\
66.35\end{array}$ & $\begin{array}{l}59.81- \\
66.71\end{array}$ & & $\begin{array}{l}53.76- \\
65.16\end{array}$ & $\begin{array}{l}59.26- \\
66.50\end{array}$ & \\
\hline \multicolumn{10}{|l|}{$\begin{array}{l}\text { Number of } \\
\text { SAIR (n) }\end{array}$} \\
\hline Mean \pm SD & $\begin{array}{l}16.71 \pm \\
5.72\end{array}$ & $\begin{array}{l}17.55 \\
\pm 5.20\end{array}$ & 0.56 & $\begin{array}{l}18.21 \pm \\
7.12\end{array}$ & $\begin{array}{l}16.61 \\
\pm 4.36\end{array}$ & 0.30 & $\begin{array}{l}16.58 \pm \\
5.85\end{array}$ & $\begin{array}{l}17.29 \\
\pm 5.37\end{array}$ & 0.69 \\
\hline $95 \% \mathrm{Cl}$ & $\begin{array}{l}14.50- \\
18.93\end{array}$ & $\begin{array}{l}15.57- \\
19.53\end{array}$ & & $\begin{array}{l}14.78- \\
21.64\end{array}$ & $\begin{array}{l}15.17- \\
18.04\end{array}$ & & $\begin{array}{l}12.86- \\
20.30\end{array}$ & $\begin{array}{l}15.68- \\
18.90\end{array}$ & \\
\hline
\end{tabular}

*: continuity correction

PVR: Post-Voiding Residual

GMFCS: Gross Motor Function Classification System

GMFM - 66: Gross Motor Function Measurement - 66

SAIR: Sphincter-Associated Input Roots (Rootlets) 


\begin{tabular}{|c|c|c|c|c|c|c|c|c|c|}
\hline \multirow[t]{2}{*}{ Characteristics } & \multicolumn{3}{|c|}{ Bladder Capacity } & \multicolumn{3}{|c|}{ Detrusor activity } & \multicolumn{3}{|l|}{ PVR } \\
\hline & Decreased & Normal & $\begin{array}{l}p \\
\text { value }\end{array}$ & Overactivity & Normal & $\begin{array}{l}p \\
\text { value }\end{array}$ & Increased & Normal & $\begin{array}{l}p \\
\text { value }\end{array}$ \\
\hline \multicolumn{10}{|l|}{$\begin{array}{l}\text { Average } \\
\text { threshold of } \\
\text { SAIR (mA) }\end{array}$} \\
\hline Median & 0.47 & 0.54 & 0.63 & 0.47 & 0.51 & 0.46 & 0.86 & 0.46 & 0.01 \\
\hline$(\mathrm{Q} 1, \mathrm{Q})$ & $\begin{array}{l}(0.37 \\
0.72)\end{array}$ & $\begin{array}{l}(0.33, \\
1.09)^{\prime}\end{array}$ & & $(0.38,1.07)$ & $\begin{array}{l}(0.32, \\
0.75)\end{array}$ & & $\begin{array}{l}(0.52, \\
1.42)\end{array}$ & $\begin{array}{l}(0.35, \\
0.67)\end{array}$ & \\
\hline $95 \% \mathrm{Cl}$ & $0.38-0.64$ & $\begin{array}{l}0.36- \\
0.80\end{array}$ & & $0.38-1.07$ & $\begin{array}{l}0.38- \\
0.64\end{array}$ & & $\begin{array}{l}0.50- \\
1.43\end{array}$ & $\begin{array}{l}0.38- \\
0.55\end{array}$ & \\
\hline \multicolumn{10}{|c|}{ *: continuity correction } \\
\hline \multicolumn{10}{|c|}{ PVR: Post-Voiding Residual } \\
\hline \multicolumn{10}{|c|}{ GMFCS: Gross Motor Function Classification System } \\
\hline \multicolumn{10}{|c|}{ GMFM - 66: Gross Motor Function Measurement - 66} \\
\hline SAIR: Sphincter- & ssociated Inp & ut Roots ( & ootlets) & & & & & & \\
\hline
\end{tabular}

Detrusor overactivity (DO) was observed in 19 (33.33\%) cases with their involuntary detrusor pressure measured over 15 $\mathrm{cm} \mathrm{H}_{2} \mathrm{O}$ in filling phase during UDS test. It turned out that non-ambulatory cases had more chance of having DO than those ambulatory ones (Table $2, p<0.05$ ). Statistical analysis showed that patients younger than 4.75 years old tended to have more chance having DO (cut-off point got from ROC curve with AUC equaling 0.68 , Figure $3-B, p<0.05$ ).

Increased PVR was seen in 12 (21.05\%) cases with their PVR ranged from 20 to $139 \mathrm{ml}$. The rest of 45 patients had PVR of less than $10 \mathrm{ml}$. Statistical comparisons were listed in Table 2. Intra-operative neurophysiological data shown that median threshold of SAIR was $0.5 \mathrm{~mA}(\mathrm{Q} 1, \mathrm{Q} 3: 0.36,0.78 \mathrm{~mA})$ in our 57 cases. It was much greater in cases with increased PVR than those with normal residual (median: 0.86 vs. $0.46 \mathrm{~mA}, p<0.01$ ). Our data demonstrated that cases in which average threshold of SAIR over $0.72 \mathrm{~mA}$ were more likely to have increased residual after voiding (cut-off value got from ROC curve with AUC equaling 0.73 , Figure $3-C, p<0.05$ ).

Bladder compliance with a mean of $29.29 \pm 12.95 \mathrm{ml} / \mathrm{cm} \mathrm{H} 2 \mathrm{O}$ (ranged from 15.00 to $65.67 \mathrm{ml} / \mathrm{cm} \mathrm{H}_{2} \mathrm{O}$ ) was observed in cases included, which turned out to all be in normal range.

\section{Discussion}

It has been reported that patients with spastic $\mathrm{CP}$ are commonly having dysfunctional urinary symptoms, such as urge urinary incontinence, frequency, urgency and others ${ }^{17-19}$. Prevalence of LUTS among our 57 cases in the current study was $52.63 \%$ (30 cases), in which 18 had multiple presentations. The results were similar to those previous studies ${ }^{20}$. In these 30 cases with LUTS, 25 showed abnormal UDS performance. Even in other 27 cases without presentation of LUTS, $15(26.32 \%)$ of them had abnormal results in UDS. Our results tied well with previous studies wherein cases with $\mathrm{CP}$ might exhibit silent bladder dysfunction even without demonstrating LUTS ${ }^{20}$. These findings suggested the importance of UDS in detecting the potential voiding dysfunction in patients with spastic CP.

The most common results of UDS in patients with spastic CP was decreased bladder capacity ${ }^{3,21}$. In the current study, nearly $50 \%$ of cases displayed a bladder capacity less than $65 \%$ of EBC, which was comparable with previous studies 22 . 
It is speculated that parents reduced the intake of water in this group of patients to lower the burden of daily care ${ }^{23}$. The presence of overactive bladder was not an uncommon finding in this population ${ }^{4,19}$. UDS in our cohort had verified similar results that DO occurred in 19 cases (33.33\%). We speculated that DO might be resulted from lack of inhibition from upper motor neurons, which leads to an overactivity of lower motor neurons ${ }^{3}$. Interestingly, we found that 11 out of 19 (57.89\%) cases with DO also have decreased bladder capacity. We assumed that DO might impact the bladder distention in an evolved bladder in their childhood in these patients, eventually causing reduced bladder capacity. Statistical comparison showed that cases with more impaired motor function have higher rate of having DO and reduced bladder capacity than those less. This might be attributed to more severe brain damage. In addition, our data showed that younger children tended to be more likely having DO than older ones. Possible explanation is the immaturity of a developing nervous system ${ }^{24}$.

Bladder compliance is a rather complicated issue in pediatric patients, as the value varies with age, and can be affected by the rate of bladder filling. In this study we took the cut-off point of $15 \mathrm{ml} / \mathrm{cm} \mathrm{H}_{2} \mathrm{O}$ and an overall normal compliance was explored. The data were in accordance with findings previously documented ${ }^{12,19}$. Nonetheless, some other researchers showed a rather reduced detrusor compliance in patients with $\mathrm{CP}^{3,18}$. The cause of different results was still unclear. We speculated that sample selection might affect the result. Further study might be needed to clarify this issue.

Previous studies have shown that SDR might have the possibility of causing bladder/bowel dysfunction after surgery ${ }^{7}$. Out of concern about such potential complication, pre-operative UDS has been conducted as a routine examination for all children with spastic CP in our center since early 2020. Based on rhizotomy protocol applied in our SDR, we need to judge all spinal nerve roots (rootlets) in cauda equina at L2 level with regard to their neurophysiological characteristics using trigger electromyography (EMG) in the surgery ${ }^{6}$. Rootlets required to be classified as input and output ones. In addition, SAIR need to be identified clearly in such procedure. Therefore, it provides the potential for us to investigate the relations between trigger EMG patterns in channel of EAS when electrically stimulating those SAIR and UDS results in these patients.

We observed that number of rootlets matching the criteria of SAIR was around 16 to 18 in average in our cases. Since SDR we performed was using single-level approach via L2, it was difficult for us to determine from which level these SAIR derived during the surgery. Interestingly, we found that in addition to those tiny sacral roots (rootlets), there are indeed many much thicker roots (rootlets) that matched the SAIR criteria. Based on our past experience, these much thicker nerve roots are generally derived from level S1 or above. Nevertheless, due to the fact that these roots (rootlets) fit the criteria, we still took them to the category of SAIR for investigation in the current study.

Due to technical difficulties, we used trigger EMG data obtained in EAS during SDR rather than detrusor and external urethral sphincter. EAS neurophysiological exploration was extrapolated to striated urethral and detrusor in this research. This point of view was raised by Sindou as the recording of EAS was easier to perform and less injury ${ }^{25}$. The monitor of EAS might give information on the micturition function by extrapolation. This is because the striated urethral and striated anal sphincter was of same somatic innervations, thus it could be postulated that such extrapolation might be logical ${ }^{26}$. In our study, we tried to minimize the difference by using average threshold value of all SAIR.

Our intra-operative neurophysiological data showed that average threshold of those SAIR was higher in 12 patients (21.05\%) with abnormal PVR (median: $0.86 \mathrm{~mA}$ vs. $0.46 \mathrm{~mA}$ ). This is a very interesting finding. Such results indicated that in cases with increased PVR, a much higher input signal was needed to induce micturition reflex. One possible explanation is that during normal urination, as the bladder volume gradually decreases, the input signal will gradually weaken until the urination reflex is completed after emptying. In the process of urination in these children, when the remaining urine in the bladder is still in an abnormal amount, the reflex stops, resulting in an abnormal amount of 
remaining urine. In addition, we found that in 17 cases with constipation, the average threshold of SAIR was significantly higher than that of patients without (median: $0.78 \mathrm{~mA}$ vs. $0.47 \mathrm{~mA}$ ). This manifestation might also be attributed to the same mechanism.

Limitations of this study included several aspects. Besides to the absence of video fluoroscopy (limited device in our center), data of EMG of pelvic floor was not be able to obtain in the current study. Difference might still exist despite the overlap of neuronal circuits innervations between EAS and bladder, which might impact the accuracy of our conclusions. Nonetheless, correlation between trigger EMG data obtained during SDR and UDS results might provide a novel approach for researchers to investigate the mechanisms beneath those clinical presentations and UDS results. Secondly, the compound muscle action potential of EAS could hardly precisely reach at $20 \mu \mathrm{V}$ during SDR procedure. Thus, the threshold of a certain SAIR might have an error. Considering that its average value was used for statistical analysis in the current study, this type of error might be still within an acceptable range. Thirdly, we did not assess the intelligence quotient in our patients. According to related articles, CP patients with lower IQ might have worse bladder function ${ }^{27}$, which was not discussed in this study. Last but not least, we only performed UDS in patients who accepted SDR, which might not be able to represent the population of spastic CP. Nonetheless, it still offered us the information of urinary dysfunction in part of these patients, thus raising the attention in bladder function of spastic CP.

\section{Conclusion}

Abnormal UDS results were prevalent in pediatric patients with spastic CP. UDS was recommended in cases with spastic $\mathrm{CP}$ due to its ability to detect silent bladder dysfunction. Motor function level, age and average trigger EMG threshold of sphincter-associated input spinal nerve roots (rootlets) were related to the abnormal UDS results.

\section{Declarations}

Ethical approval The study was conducted in accordance with the principles of the Declaration of Helsinki, and the study protocol was approved by the ethics committee of Shanghai Children's Hospital. Because of the retrospective nature of the study, patient consent for inclusion was waived.

Availability of data and materials Data used to support the findings of this study were included within the article.

Competing interests No financial or non-financial benefits have been received or will be received from any party related directly or indirectly to the subject of this article.

Funding Shanghai Children's Hospital (2020YGZQ05), Shanghai Jiao Tong University (YG2021QN117).

Authors' contributions All authors have read and approved the manuscript. JWB conceptualized and designed the study, interpreted the data, and critically revised the manuscript. XB and GBJ revised the manuscript. SHZ and XB interpreted the data. ZQJ, WM, LS and CF helped designed the study and interpreted the data.

\section{References}

1. Drzewiecki B, Bauer S. Urodynamic testing in children: indications, technique, interpretation and significance. The Journal of urology. 2011;186(4):1190-1197.

2. Cotter K, Levy M, Goldfarb R, et al. Urodynamic Findings in Adults With Moderate to Severe Cerebral Palsy. Urology. 2016;95:216-221. 
3. Karaman M, Kaya C, Caskurlu T, et al. Urodynamic findings in children with cerebral palsy. International journal of urology : official journal of the Japanese Urological Association. 2005;12(8):717-720.

4. Aravamuthan B, Fehlings D, Shetty S, et al. Variability in Cerebral Palsy Diagnosis. Pediatrics. 2021;147(2).

5. Surveillance of cerebral palsy in Europe: a collaboration of cerebral palsy surveys and registers. Surveillance of Cerebral Palsy in Europe (SCPE). Developmental medicine and child neurology. 2000;42(12):816-824.

6. Zhan Q, Tang L, Wang Y, et al. Feasibility and effectiveness of a newly modified protocol-guided selective dorsal rhizotomy via single-level approach to treat spastic hemiplegia in pediatric cases with cerebral palsy. Child's nervous system : ChNS : official journal of the International Society for Pediatric Neurosurgery. 2019;35(11):2171-2178.

7. Steinbok P, Schrag C. Complications after selective posterior rhizotomy for spasticity in children with cerebral palsy. Pediatric neurosurgery. 1998;28(6):300-313.

8. Morris C, Bartlett D. Gross Motor Function Classification System: impact and utility. Developmental medicine and child neurology. 2004;46(1):60-65.

9. Alotaibi M, Long T, Kennedy E, et al. The efficacy of GMFM-88 and GMFM-66 to detect changes in gross motor function in children with cerebral palsy (CP): a literature review. Disability and rehabilitation. 2014;36(8):617-627.

10. Bauer S, Nijman R, Drzewiecki B, et al. International Children's Continence Society standardization report on urodynamic studies of the lower urinary tract in children. Neurourology and urodynamics. 2015;34(7):640-647.

11. Hjälmås K. Urodynamics in normal infants and children. Scandinavian journal of urology and nephrology. Supplementum. 1988;114:20-27.

12. Richardson I, Palmer L. Clinical and urodynamic spectrum of bladder function in cerebral palsy. The Journal of urology. 2009;182:1945-1948.

13. Nevéus T, von Gontard A, Hoebeke P, et al. The standardization of terminology of lower urinary tract function in children and adolescents: report from the Standardisation Committee of the International Children's Continence Society. The Journal of urology. 2006;176(1):314-324.

14. Chang S, Chiang I, Hsieh C, et al. Age- and gender-specific nomograms for single and dual post-void residual urine in healthy children. Neurourology and urodynamics. 2013;32(7):1014-1018.

15. Shin J, Park C, Kim H, et al. Significance of low compliance bladder in cauda equina injury. Spinal cord. 2002;40(12):650-655.

16. Zhan Q, Yu X, Jiang W, et al. Whether the newly modified rhizotomy protocol is applicable to guide single-level approach SDR to treat spastic quadriplegia and diplegia in pediatric patients with cerebral palsy? Child's nervous system : ChNS : official journal of the International Society for Pediatric Neurosurgery. 2020;36(9):1935-1943.

17. Gündoğdu G, Kömür M, Avlan D, et al. Relationship of bladder dysfunction with upper urinary tract deterioration in cerebral palsy. Journal of pediatric urology. 2013;9(5):659-664.

18. Bross S, Honeck P, Kwon S, et al. Correlation between motor function and lower urinary tract dysfunction in patients with infantile cerebral palsy. Neurourology and urodynamics. 2007;26(2):222-227. 
19. Chiu P, Yam K, Lam T, et al. Does selective dorsal rhizotomy improve bladder function in children with cerebral palsy? International urology and nephrology. 2014;46(10):1929-1933.

20. Samijn B, Van Laecke E, Renson C, et al. Lower urinary tract symptoms and urodynamic findings in children and adults with cerebral palsy: A systematic review. Neurourology and urodynamics. 2017;36(3):541-549.

21. Ersoz M, Kaya K, Erol S, et al. Noninvasive evaluation of lower urinary tract function in children with cerebral palsy. American journal of physical medicine \& rehabilitation. 2009;88(9):735-741.

22. Tandr Y, Ozgen MB, Ozek M, et al. Urodynamic Findings in Children with Cerebral Palsy Prior to Dorsal Rhizotomy. 2020. J Urol Surg. 2021;8(1):33-39.

23. Van Laecke E, Raes A, Vande Walle J, et al. Adequate fluid intake, urinary incontinence, and physical and/or intellectual disability. The Journal of urology. 2009;182:2079-2084.

24. Wright A, Fletcher O, Scrutton D, et al. Bladder and bowel continence in bilateral cerebral palsy: A population study. Journal of pediatric urology. 2016;12(6):383.e381-383.e388.

25. Sindou M, Joud A, Georgoulis G. Usefulness of external anal sphincter EMG recording for intraoperative neuromonitoring of the sacral roots-a prospective study in dorsal rhizotomy. Acta neurochirurgica. 2021;163(2):479-487.

26. James $\mathrm{H}$, Mulcahy J, Walsh J, et al. Use of anal sphincter electromyography during operations on the conus medullaris and sacral nerve roots. Neurosurgery. 1979;4(6):521-523.

27. Samijn Bieke,Van den Broeck Christine,Deschepper Ellen et al. Risk Factors for Daytime or Combined Incontinence in Children with Cerebral Palsy. J Urol. 2017, 198: 937-943.

\section{Figures}

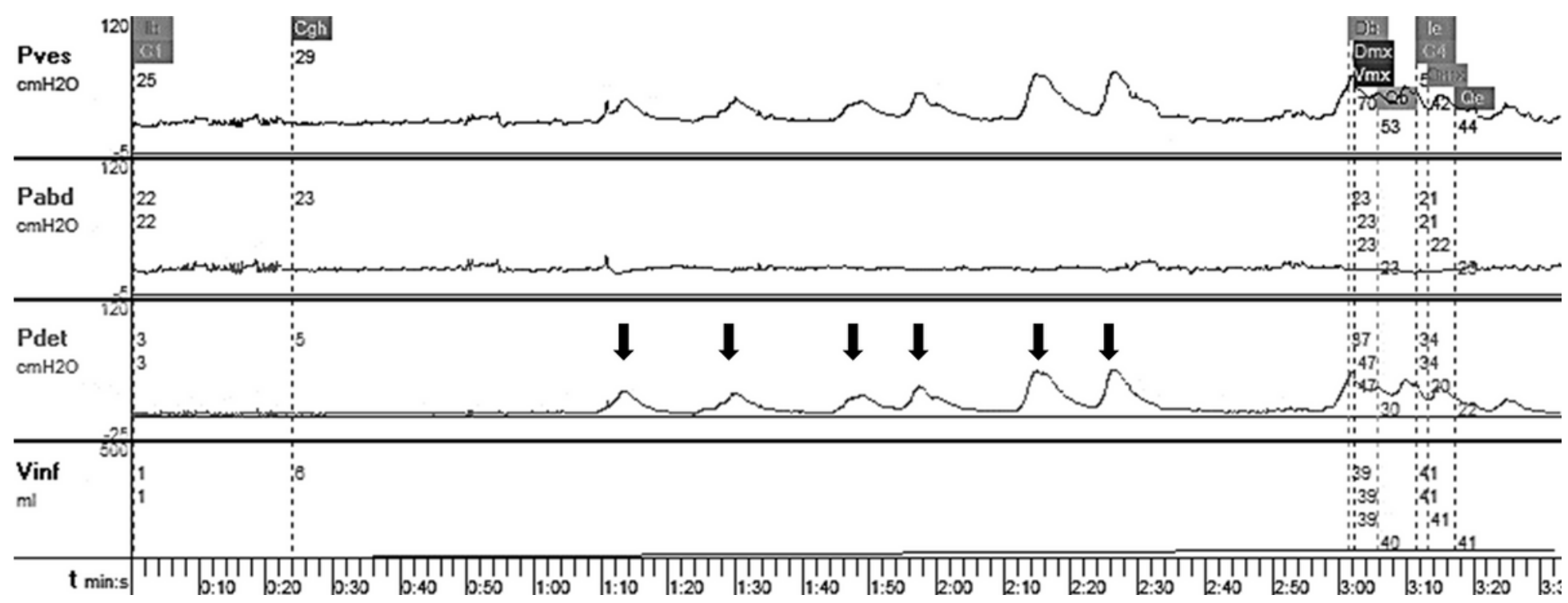

Figure 1

Representative urodynamic result of spastic cerebral palsy showing involuntary detrusor overactivity during filling phase. Pdet $=$ Pves - Pabd. X-axis, time; Y-axis, pressure $(\mathrm{cm} \mathrm{H2O})$. Arrows: non-voiding contractions. 


\section{External Anal Sphincter $0.48 \mathrm{~mA}, 23 \mu \mathrm{V}$}

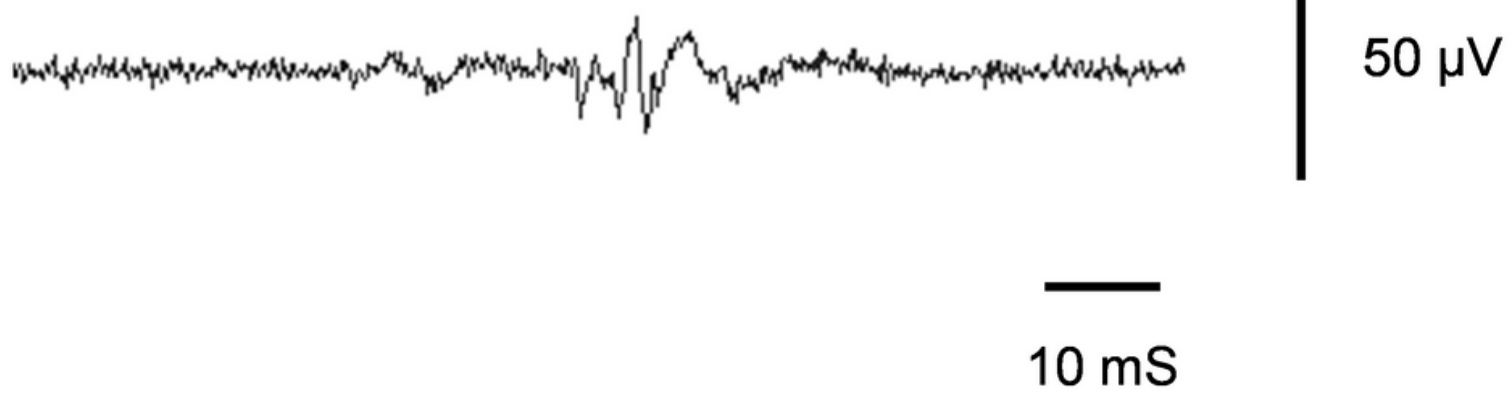

Figure 2

Intraoperative trigger electromyography during surgery. The freezing screen showed a representative external anal sphincter activity elicited by electrically stimulating a dorsal rootlet. This stimulated rootlet is defined as sphincterassociated input rootlet (SAIR).

A

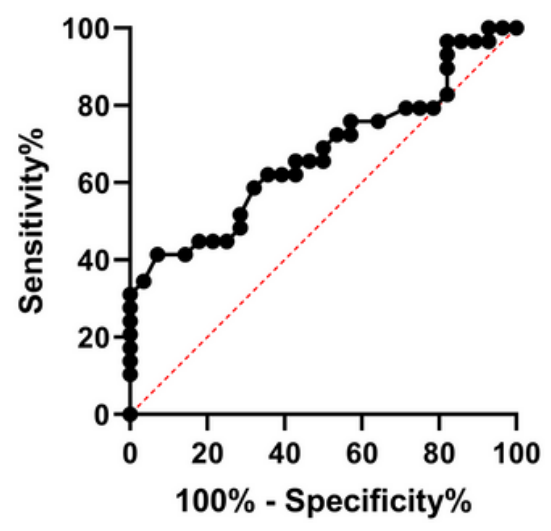

B

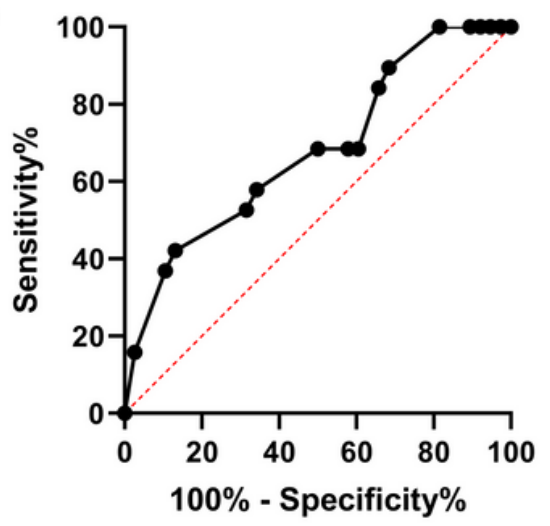

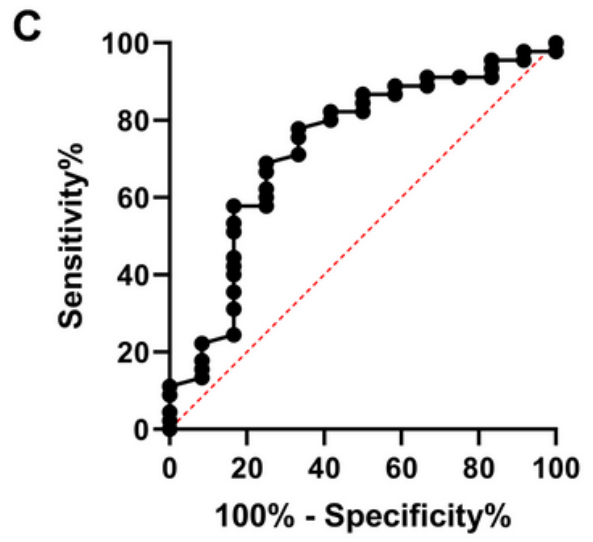

Figure 3

(A) ROC curve of GMFM - 66 scores in cases with reduced bladder capacity (AUC: 0.67; Cl: 0.53 - 0.81; p=0.03; cut-off point: 70.22 , sensitivity: $41.38 \%$, specificity: $92.86 \%$ ). (B) ROC curve of age in cases with DO (AUC: 0.68; Cl: 0.53-0.83; $p=0.03$; cut-off point: 4.75 years old, sensitivity: $42.11 \%$, specificity: $86.84 \%$ ). (C) ROC curve of SAIR threshold in cases with elevated PVR (AUC: 0.73; Cl: 0.56-0.90; p=0.02; cut-off point: $0.72 \mathrm{~mA}$, sensitivity: $77.78 \%$, specificity: $66.67 \%$ ). ROCヌReceiver Operating Characteristic 